\title{
Directional synthetic aperture flow imaging
}

\author{
Jensen, Jørgen Arendt; Nikolov, Svetoslav
}

Published in:

I E E E Transactions on Ultrasonics, Ferroelectrics and Frequency Control

Link to article, DOI:

10.1109/TUFFC.2004.1334843

Publication date:

2004

Document Version

Publisher's PDF, also known as Version of record

Link back to DTU Orbit

Citation (APA):

Jensen, J. A., \& Nikolov, S. (2004). Directional synthetic aperture flow imaging. I E E E Transactions on Ultrasonics, Ferroelectrics and Frequency Control, 51(9), 1107-1118.

https://doi.org/10.1109/TUFFC.2004.1334843

\section{General rights}

Copyright and moral rights for the publications made accessible in the public portal are retained by the authors and/or other copyright owners and it is a condition of accessing publications that users recognise and abide by the legal requirements associated with these rights.

- Users may download and print one copy of any publication from the public portal for the purpose of private study or research.

- You may not further distribute the material or use it for any profit-making activity or commercial gain

- You may freely distribute the URL identifying the publication in the public portal

If you believe that this document breaches copyright please contact us providing details, and we will remove access to the work immediately and investigate your claim. 


\title{
Directional Synthetic Aperture Flow Imaging
}

\author{
Jørgen Arendt Jensen, Senior Member, IEEE and Svetoslav Ivanov Nikolov
}

\begin{abstract}
A method for flow estimation using synthetic aperture imaging and focusing along the flow direction is presented. The method can find the correct velocity magnitude for any flow angle, and full color flow images can be measured using only 32 to 128 pulse emissions. The approach uses spherical wave emissions with a number of defocused elements and a linear frequency-modulated pulse (chirp) to improve the signal-to-noise ratio. The received signals are dynamically focused along the flow direction and these signals are used in a cross-correlation estimator for finding the velocity magnitude. The flow angle is manually determined from the B-mode image. The approach can be used for both tissue and blood velocity determination. The approach was investigated using both simulations and a flow system with a laminar flow. The flow profile was measured with a commercial 7.5 MHz linear array transducer. A plastic tube with an internal diameter of $17 \mathrm{~mm}$ was used with an EcoWatt 1 pump generating a laminar, stationary flow. The velocity profile was measured for flow angles of 90 and 60 degrees. The RASMUS research scanner was used for acquiring radio frequency ( $\mathrm{RF}$ ) data from 128 elements of the array, using 8 emissions with 11 elements in each emission. A 20- $\mu$ s chirp was used during emission. The RF data were subsequently beamformed off-line and stationary echo canceling was performed. The 60-degree flow with a peak velocity of $0.15 \mathrm{~m} / \mathrm{s}$ was determined using 16 groups of 8 emissions, and the relative standard deviation was $0.36 \%$ $(0.65 \mathrm{~mm} / \mathrm{s})$. Using the same setup for purely transverse flow gave a standard deviation of $1.2 \%(2.1 \mathrm{~mm} / \mathrm{s})$. Variation of the different parameters revealed the sensitivity to number of lines, angle deviations, length of correlation interval, and sampling interval. An in vivo image of the carotid artery and jugular vein of a healthy 29-year-old volunteer was acquired. A full color flow image using only 128 emissions could be made with a high-velocity precision.
\end{abstract}

\section{INTRODUCTION}

$\mathrm{C}$ UURRENT ultrasound systems measure the blood flow by emitting pulses in one direction a number of times. The velocity is then found by segmenting the signals and correlating these segments to find either the phase shift or the displacement between signals and thereby the velocity [1]-[3]. This technique has several problems: Foremost, the frame rate is directly dependent on the number of image directions in which the flow is determined. Normally 8 to 16 emissions are used per direction for a total of 50 to 100 directions. For a pulse repetition frequency of $5 \mathrm{kHz}$, the

Manuscript received March 5, 2003; accepted May 5, 2004. This work was supported by the grants 9700883 and 9700563 from the Danish Science Foundation and by B-K Medical A/S, Herlev, Denmark.

The authors are with the Center for Fast Ultrasound Imaging, Ørsted•DTU, Bldg. 348, Technical University of Denmark, DK-2800 Kgs. Lyngby, Denmark (e-mail: jaj@oersted.dtu.dk). frame rate is then 3 images/sec in the worst case. This is too low to adequately sample the pulsatility of the flow. Secondly, only a few lines are used in the estimation. This makes the standard deviation quite high, and it is often 10 to $20 \%$ in clinical situations. Furthermore, only the velocity component projected onto the ultrasound beam direction is found. Often the velocity cannot be determined, since many vessels run along the skin surface. This can be solved by mechanically tilting the transducer, but this often squeezes the vessel and alters the flow. A second approach tilts the emitted beam to visualize a transverse flow, but then other flow directions at, e.g., bifurcations are transverse to the emitted beam and are thereby invisible to the flow estimator.

Several authors have addressed the transverse velocity problem. Fox [4] suggested using two crossing beams for estimating the velocity vector. The approach, however, needs a large transducer especially for large investigation depths, which makes it cumbersome in the clinic, and the standard deviation rises with depth. Trahey et al. [5] used speckle tracking to search for the displacement in two directions. This necessitates a very fast image acquisition and makes the color flow resolution low. Bonnefous [6] suggested using parallel beamformation to make a transverse beam. This approach unfortunately breaks down when the flow angle is not 90 degrees. Jensen and Munk [7], [8] and Anderson [9] suggested using a transverse beam modulation to introduce an oscillation transverse to the beam direction to generate a signal suitable for transverse velocity estimation. The approach uses 2 or 3 parallel beamformers and a special estimator [10] to find the velocity vector. Newhouse and co-workers [11] suggested using the bandwidth of the received signal as an indication of transverse velocity. None of the techniques have yet been implemented in clinical scanners, and they still have to show their clinical efficacy.

The frame rate and image size can be decoupled by using the synthetic aperture flow method suggested in [12], [13], where continuous data can be acquired simultaneously in all directions of the image. The frame rate is then decoupled from the pulse repetition frequency, and very fast imaging can be attained. This also makes it possible to average over more measurements for each estimate to improve the standard deviation of the estimates. The time for averaging is, thus, limited only by the stationarity of the flow corresponding to the duration over which the flow essentially has the same velocity. The third problem mentioned above can be solved by using directional focusing along the flow direction as suggested in [14], [15]. Here the angle between the velocity vector and the ultrasound 
beam is determined from the B-mode image, and the received ultrasound signals are focused along the direction of the flow. These signals are correlated and the displacement found. From this the correct velocity magnitude can be found by dividing with the time between pulse emissions, and a flow in any direction to the ultrasound beam can be detected.

These two approaches are combined in this paper to make a color flow system that can yield a velocity image independent of the flow angle. Synthetic aperture imaging can yield a dynamically focused beam in both transmit and receive, and any point in the image can be dynamically focused. It is therefore possible to beamform the received signals in any direction, and thereby possible to focus signals along the direction of the flow. These signals can be generated continuously for all image points, and they can be used to find the displacement between two such signals, whereby the velocity along the signal direction can be found. This is the method investigated in this paper.

The theory of the approach is described in Section II, which details how image acquisition and focusing are performed; Section III describes the directional focusing and velocity estimation. The approach is investigated using simulations in Section IV and using an experimental ultrasound scanner and a flow rig in Section V. A parameter study is presented in Section VI. Full color flow images are shown in Section VII and an in vivo flow image of the carotid artery is presented in Section VIII.

\section{Directional Synthetic Aperture Flow EstimATION}

A synthetic aperture (SA) image is acquired by emitting spherical waves from the ultrasound transducer and then receiving signals on the individual elements. Since the position of emission is known, the precise time from emission to reception can be calculated and used in the focusing. The distance from the source of a spherical wave to a point in the image and back to the receiving element is given by:

$$
d\left(\vec{r}_{p}, \vec{r}_{t}, \vec{r}_{r}\right)=\left|\vec{r}_{p}-\vec{r}_{t}\right|+\left|\vec{r}_{p}-\vec{r}_{r}\right|
$$

where $\vec{r}_{p}$ is the point in the image, $\vec{r}_{t}$ is the location of the transmission, and $\vec{r}_{r}$ is the position of the receiving element. Focusing for a single transmission is then performed by adding the received signals $g(t, i)$ from all elements in phase; $\vec{r}_{r}(i)$ is the position of the $i$-th receive element. The focused signal is then

$$
L_{j}\left(\vec{r}_{p}\right)=\sum_{i=1}^{N_{r}} g\left(\frac{d\left(\vec{r}_{p}, \vec{r}_{t}(j), \vec{r}_{r}(i)\right)}{c}, i\right),
$$

where $c$ is the speed of sound, $i$ is the receive element number, $j$ is the transmitting element number, and $N_{r}$ is the number of receiving elements. The data for this image point are now aligned for the particular emission and

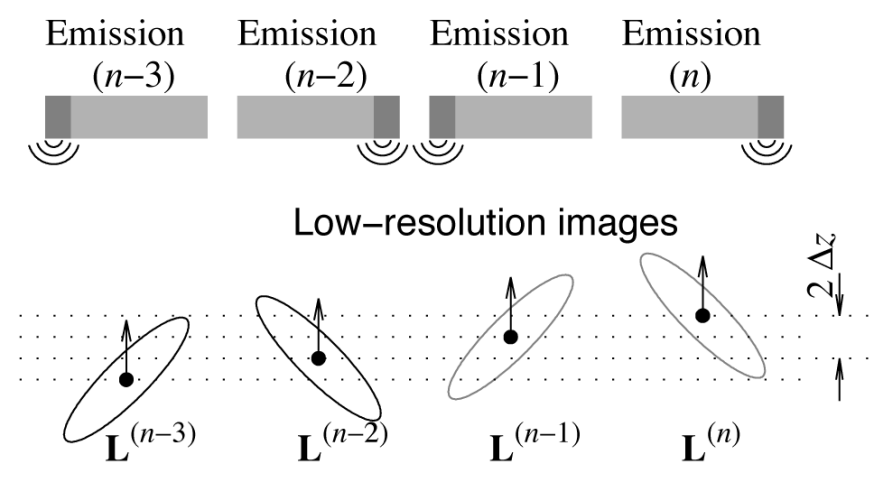

\section{High-resolution images}
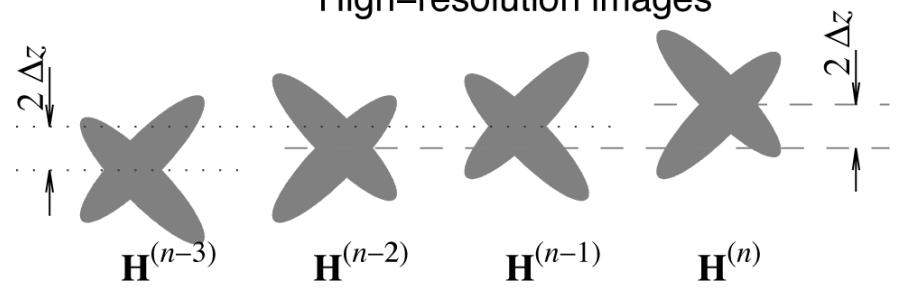

Fig. 1. Beamformation of high-resolution images from the lowresolution images using two emissions for a moving scatterer (from [12]). The top drawing shows the emitting element in the aperture for the different time instances. The corresponding low-resolution images are shown below. The high-resolution images shown at the bottom are made from the last two low-resolution images.

receiving elements, and the synthetic transmit focusing is therefore obtained by adding the signals for all emissions as

$$
H\left(\vec{r}_{p}\right)=\sum_{j=1}^{N_{t}} L_{j}\left(\vec{r}_{p}\right)
$$

to give the best possible focused image. Here $N_{t}$ is the number of transmitting events, and $H\left(\vec{r}_{p}\right)$ is denoted a high-resolution image. The advantage of this approach is that the image is dynamically focused in both transmit and receive at all locations.

Since the image is constructed over a number of emissions, the signal from a moving object will not be at the same position for the individual emissions and will therefore not be summed coherently. It has been thought that this will make SA flow estimation impossible, but the problem can be circumvented. For velocity estimation it is necessary to compare two measurements, and then find the positional shift between the measurements. The vital demand is that the two measurements are obtained in exactly the same way, so that the difference is only the movement between measurements. This was used in [12] to devise an SA method, that can be used for flow estimation.

The approach is illustrated in Fig. 1. Two elements at the edges of the transducer are used for emission, and the signals from a single-point scatterer are received by all transducer elements after each emission. The corresponding point-spread function (PSF) for the low-resolution images are shown in the middle graph. They are in general tilted toward the transmitting element. The point scatterer moves toward the receiving aperture with a velocity 


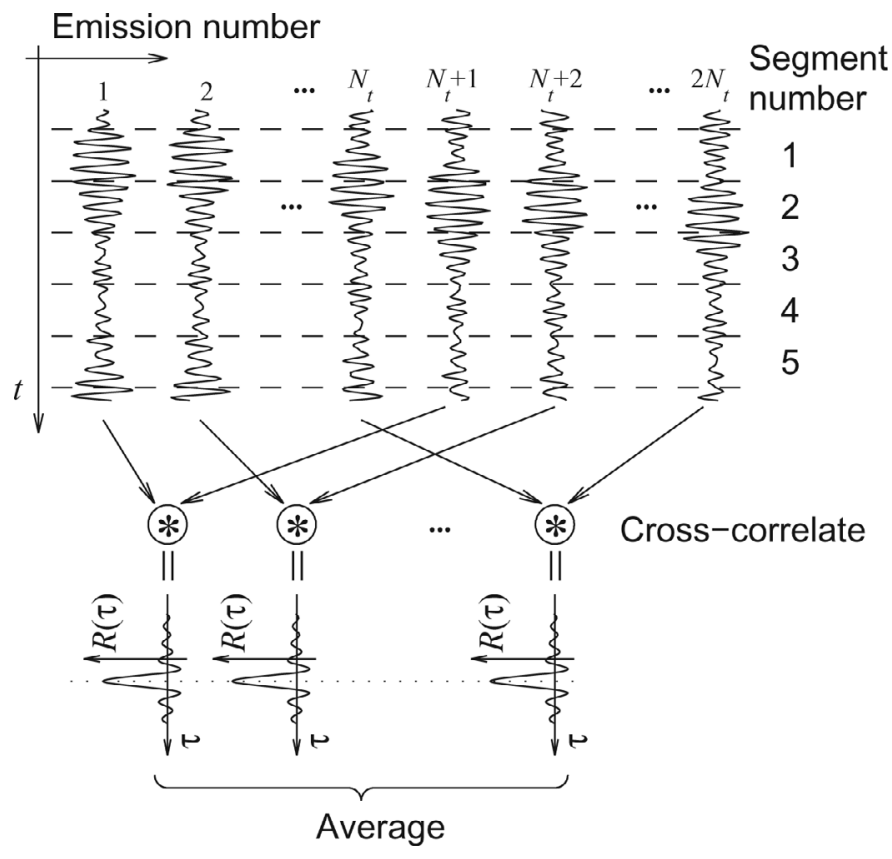

Fig. 2. Creation of the cross-correlation function and its averaging over several emissions (adapted from [12]). The top graph shows the high-resolution RF data after beamformation for several emissions. The emission sequence is repeated after $N_{t}$ emissions, and data for the same emission sequence can be correlated.

of $v_{z}$ and travels a distance of $\Delta z=v_{z} T_{p r f}$ between pulse emissions, where $T_{p r f}=1 / f_{p r f}$ is time between emissions. The variable $n$ denotes the absolute number for the emission. High-resolution images can be made by combining data from emission $[(n-3),(n-2)],[(n-2),(n-1)]$ and $[(n-1),(n)]$. It is noted that the shape of the PSF when using $[(n-3),(n-2)]$ and $[(n-1),(n)]$ is the same, apart from the translation in the axial direction. The motion can therefore be found by cross-correlating signals from the two high-resolution images and then determining the shift in position of the correlation peak. Dividing by the time between the two images gives the velocity. It should be noted that the image created from $[(n-2),(n-1)]$ can be used and correlated with $[(n),(n+1)]$, so that a new usable high-resolution image is created after every emission. These images can be created using recursive ultrasound imaging [16], where the oldest emission is replaced by the newest emission to yield a frame rate equal to the pulse repetition frequency. The current high-resolution image is, thus, made from the last $N_{t}$ emissions, where the order of transmissions for the low-resolution images changes. The first has a sequence of emission of $\left[1,2,3, \ldots N_{t}\right]$, the second $\left[2,3, \ldots N_{t}, 1\right]$, then $\left[3, \ldots N_{t}, 1,2\right]$ and so forth.

The cross-correlation function can be averaged over all the high-resolution image pairs, since the shift between the pairs is the same for a constant velocity. This is shown in Fig. 2, where the beamformed signals from one direction in the high-resolution image is shown as a function of emission number. The high-resolution images are generated from a sequence of $N_{t}$ low-resolution images, which is repeated twice. Data for emission 1 and $N_{t}+1$ are therefore

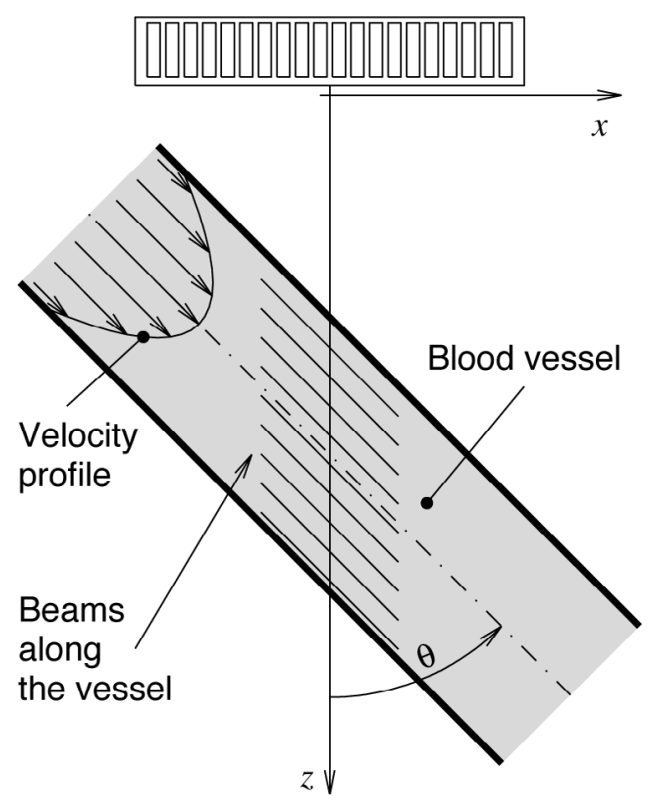

Fig. 3. The directional signals shown inside the vessel are focused along the flow vector direction.

generated by exactly the same sequence of transmissions and can be correlated. The same is true for 2 and $N_{t}+2$, 3 and $N_{t}+3$ and so forth. The signals are divided into segments to yield the velocity as a function of depth, and segments from the same depth are correlated as described above. The shift in the position of the maximum in the cross-correlation function will be the same for all correlation pairs, if the velocity is constant as is indicated in the lower part of Fig. 2. The cross-correlation functions can therefore be averaged to obtain a better estimate.

It should be noted that data are continuously available from all places in the image, since a high-resolution image can be made of the whole region after each pulse emission. This improves stationary echo canceling, as the initialization of filters is less critical when using continuous data. The velocity estimates can also be averaged over many more emissions than in a traditional system, as long as the velocity is roughly constant over the time of averaging.

\section{Directional Velocity Estimation}

In a traditional ultrasound scanner the received signal is focused along the direction of the emitted beam. This is not necessary in an SA system, since data are available from all directions of the image simultaneously. The beams can therefore be formed in any order and direction, and it is possible to beamform signals along the direction of the flow as suggested in [14] and [15]. The receive focus points are placed along lines parallel to the flow direction as shown in Fig. 3 to track the movement of the scatterers.

The focusing points are given by

$$
\vec{r}_{p}(k)=\left[k \Delta x^{\prime} \sin (\theta)+x_{s t}, 0, k \Delta x^{\prime} \cos (\theta)+z_{s t}\right],
$$


where $\Delta x^{\prime}$ is the spatial sampling interval, $k$ is the sample index, $\theta$ is the angle between the flow vector and the $z$ axis, and $\left(x_{s t}, 0, z_{s t}\right)$ is the point in the image for velocity estimation. The value of $k$ is then from $-N_{k} / 2$ to $N_{k} / 2$, where $N_{k}+1$ is the number of samples in one directional signal. This can be viewed as a coordinate system placed at the image point $\left(x_{s t}, 0, z_{s t}\right)$ and then rotated through an angle $\theta$ to be oriented along the flow direction. Beamforming for the values $\vec{r}_{p}(k)$ gives one directionally focused signal $y_{d}(k)$ for the given depth as:

$$
y_{d}(k)=\sum_{j=1}^{N_{t}} L_{j}\left(\vec{r}_{p}(k)\right) .
$$

For the next received directional signal, the scatterers have moved a distance

$$
\overrightarrow{d_{s}}=\vec{v} T_{p r f} N_{t}
$$

where $\vec{v}=|\vec{v}|[\sin (\theta), 0, \cos (\theta)]$ corresponding to a sample index of

$$
k_{s}=\frac{|\vec{v}| T_{p r f} N_{t}}{\Delta x} .
$$

Cross-correlating two received signals, thus, gives

$$
\begin{aligned}
R_{12}(l) & =\frac{1}{N_{k}+1} \sum_{k=-N_{k} / 2}^{N_{k} / 2} y_{d}^{(n)}(k) y_{d}^{\left(n+N_{t}\right)}(k+l) \\
& =\frac{1}{N_{k}+1} \sum_{k=-N_{k} / 2}^{N_{k} / 2} y_{d}^{(n)}(k) y_{d}^{(n)}\left(k+l-k_{s}\right) \\
& =R_{11}\left(l-k_{s}\right),
\end{aligned}
$$

where $y_{d}^{(n)}(k)$ is the directional signal focused after emission $n$, and $R_{11}(l)$ is the autocorrelation function of the directional signal. A global maximum is, thus, found at $l=k_{s}$, and the velocity is determined by

$$
|\vec{v}|=\frac{k_{s} \Delta x}{T_{p r f} N_{t}} .
$$

The velocity magnitude is hereby found by the method regardless of the flow angle.

The quantization into sampling intervals in the crosscorrelation function often gives a too-coarse resolution in the velocity estimates, and a more precise estimate can be found by making an interpolation around the maximum point by employing [3]

$$
\begin{aligned}
k_{\text {int }}= & k_{s}- \\
& \frac{\hat{R}_{12}\left(k_{s}+1\right)-\hat{R}_{12}\left(k_{s}-1\right)}{2\left(\hat{R}_{12}\left(k_{s}+1\right)-2 \hat{R}_{12}\left(k_{s}\right)+\hat{R}_{12}\left(k_{s}-1\right)\right)},
\end{aligned}
$$

and the velocity is then found from

$$
|\vec{v}|=\frac{k_{i n t} \Delta x}{T_{p r f} N_{t}}
$$

The correct angle for the beamformation must be inserted into the approach. This is done by beamforming a B-mode image, and then manually determining the angle for the flow. This angle is used in subsequent beamformation of the directional signals for the flow estimation. It should be noted that different angles can be used in different parts of an image. It is therefore possible to follow the flow in a bending vessel, where the flow angle changes as a function of spatial position. An angle determination scheme as suggested in [17] can, thus, be used.

The influence of an error in the angle determination is quantified in Section VI.

\section{Simulation of Approach}

Parabolic flow in a rigid vessel has been simulated using the Field II program [18]. The peak velocity in the vessel is $0.15 \mathrm{~m} / \mathrm{s}$, its radius is $8.5 \mathrm{~mm}$, and the center is $37.5 \mathrm{~mm}$ from the transducer. The angle between the transducer's acoustical axis and the vessel is 60 degrees. The 450,000 point scatterers are propagated between pulse emissions according to their spatial velocity, and 800 consecutive pulse emissions are simulated. The received signal for all 128 receiving elements are calculated, and the signals are beamformed along the velocity direction.

The simulations were performed by using 8 emissions equally spread over the 128-element aperture, where each emission used 11 elements defocused to emulate a spherical wave emission [19], [20]. The initial spherical wave has a radius of $6 w$, where $w$ is the transducer pitch. The center of the wave is placed $6 w$ behind the transducer surface and the elements' delays $\Delta t(j)$ are given by

$$
\Delta t(j)=\frac{\left|\vec{r}_{t}-\vec{r}_{e}(j)\right|}{c}-\frac{6 w}{c},
$$

where $\vec{r}_{t}$ denotes the center of the emitted wave, and $\vec{r}_{e}(j)$ is the position of the transmitting element $j$. The transmission was repeated with $f_{p r f}=3 \mathrm{kHz}$, and 800 individual emissions were simulated by repeating the sequence 100 times. A summary of the parameters used is shown in Table I. A short pulse was used for excitation of the 11 elements. Sixteen pairs of 8 emissions were used for estimating the velocity.

The directional signals were beamformed after simulation using a fixed angle of 60 degrees input to the beamformation routine in (4). Linear interpolation between received sample values was performed in the beamformation to ensure precise focusing.

The resulting velocity profiles are shown in Fig. 4 . The top graph shows the 11 profiles estimated from the data, where 128 emissions were used for each estimate. A $50 \%$ overlap between the data for the profiles was employed to give the 11 estimates. The lower graph shows the mean over all the profiles (solid line) \pm 3 standard deviations (dashed lines) along with the true velocity profile (dashed dotted line). The results are evaluated through their bias and standard deviation. The mean bias is defined as: 
TABLE I

Standard Parameters for Transducer and Parabolic Flow Simulation.

Transducer center frequency Assumed speed of sound Wavelength

Pitch of transducer element

Height of transducer element Kerf

Number of receive elements

Number of transmit events

Number of transmitting elements

Elevation focus

Pulse repetition frequency

RF sampling frequency

Distance between estimates

Sampling interval for lines

Correlation interval

Radius of vessel

Distance to vessel center

Peak velocity in flow

\begin{tabular}{cc}
$f_{0}$ & $7 \mathrm{MHz}$ \\
$c$ & $1480 \mathrm{~m} / \mathrm{s}$ \\
$\lambda=c / f_{0}$ & $0.22 \mathrm{~mm}$ \\
$w$ & $0.208 \mathrm{~mm}$ \\
$h_{e}$ & $4.5 \mathrm{~mm}$ \\
$k_{e}$ & $0.035 \mathrm{~mm}$ \\
$N_{r}$ & 128 \\
$N_{t}$ & 8 \\
$N_{e}$ & 11 \\
$R_{e}$ & $20 \mathrm{~mm}$ \\
$f_{p r f}$ & $3 \mathrm{kHz}$ \\
$f_{s}$ & $100 \mathrm{MHz}$ \\
$d z$ & $0.25 \mathrm{~mm}$ \\
$\Delta x=\lambda / 20$ & $0.011 \mathrm{~mm}$ \\
$-10 \lambda: 10 \lambda$ & $-2.2: 2.2 \mathrm{~mm}$ \\
$R$ & $8.5 \mathrm{~mm}$ \\
$z_{s t}$ & $37.5 \mathrm{~mm}$ \\
$v_{0}$ & $0.15 \mathrm{~m} / \mathrm{s}$ \\
\hline
\end{tabular}
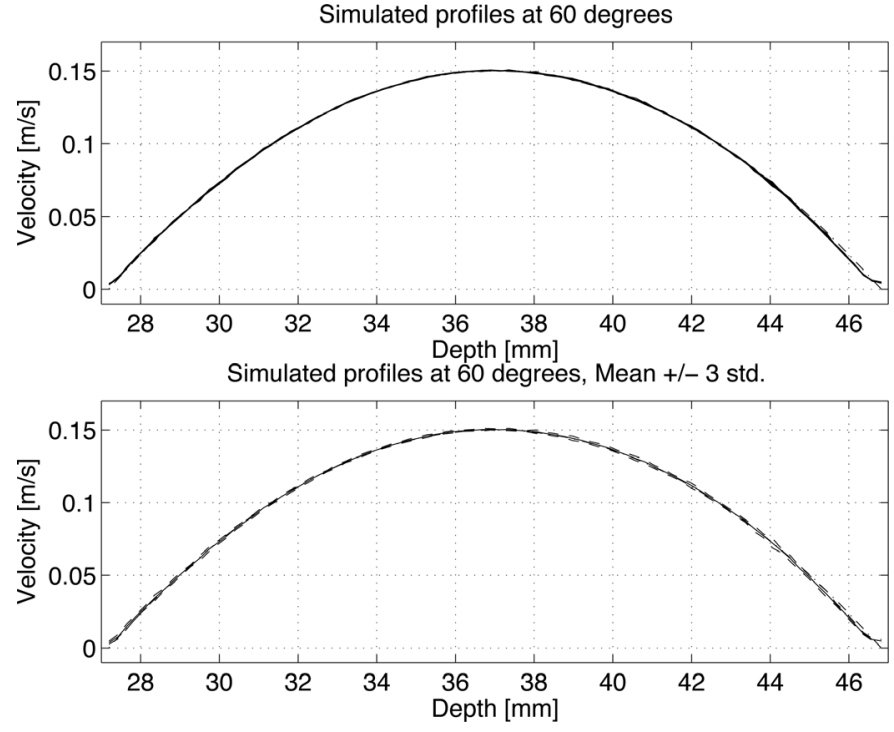

Fig. 4. Estimated profiles from simulated parabolic flow at a 60degree flow angle. The top graph shows the 11 estimated profiles and the bottom graph shows the mean profile (solid line) \pm 3 standard deviations (dashed lines).

$$
v_{\text {bias }}=\frac{1}{N_{p} N_{v}} \sum_{j=1}^{N_{p}} \sum_{k=1}^{N_{v}} \hat{v}\left(\vec{r}_{k}, j\right)-v\left(\vec{r}_{k}\right)
$$

where $N_{p}$ is the number of profiles estimated, $N_{v}$ is the number of values in one profile, $\vec{r}_{k}$ is the position for the velocity estimate, $\hat{v}\left(\vec{r}_{k}, i\right)$ is the estimated velocity for position $k$ and profile number $i$, and $v\left(\vec{r}_{k}\right)$ is the true velocity. The standard deviation is defined as:
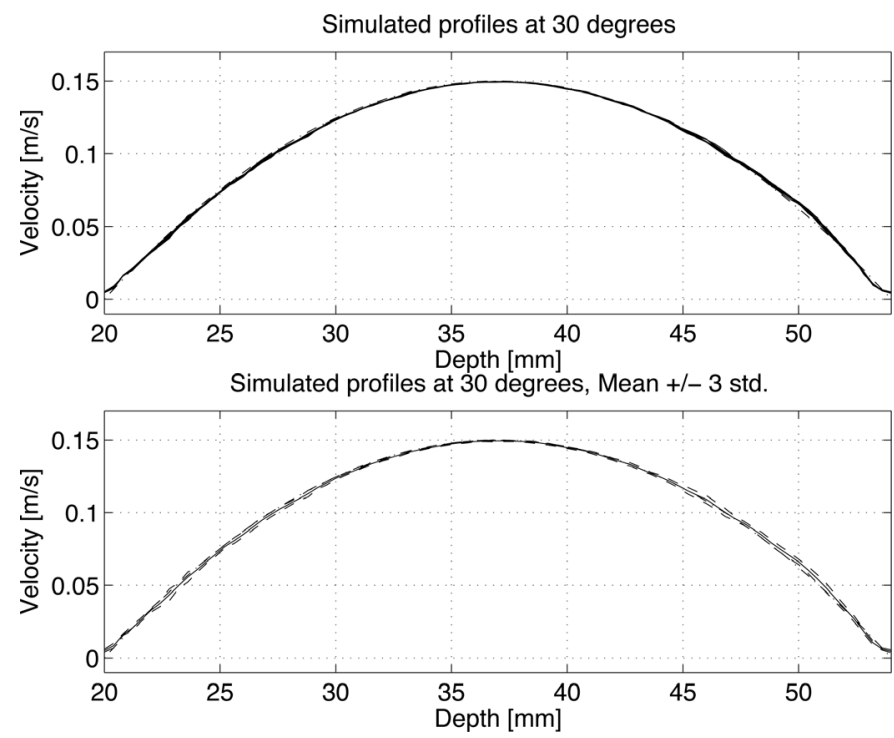

Fig. 5. Estimated profiles from simulated parabolic flow at a 30degree flow angle. The top graph shows the 11 estimated profiles and the bottom graph shows the mean profile (solid line) \pm 3 standard deviations (dashed lines).

$$
\begin{aligned}
v_{s t d} & =\sqrt{\frac{1}{N_{p} N_{v}} \sum_{j=1}^{N_{p}} \sum_{k=1}^{N_{v}}\left(\hat{v}\left(\vec{r}_{k}, j\right)-\bar{v}\left(\vec{r}_{k}\right)\right)^{2}} \\
\bar{v}\left(\vec{r}_{k}\right) & =\frac{1}{N_{p}} \sum_{j=1}^{N_{p}} \hat{v}\left(\vec{r}_{k}, j\right),
\end{aligned}
$$

where $\bar{v}\left(\vec{r}_{k}\right)$ is the mean velocity profile estimate.

The mean bias over the full profile is $-13.4 \mu \mathrm{m} / \mathrm{s}$, $(-0.0090 \%$ compared to the peak velocity in the vessel) and the mean standard deviation over the profile is $0.36 \mathrm{~mm} / \mathrm{s},(0.24 \%$ compared to the peak velocity in the vessel). Similar profiles are shown in Fig. 5 for a 30degree angle. Here the mean bias over the full profile is $-122.5 \mu \mathrm{m} / \mathrm{s},(-0.082 \%)$ and the mean standard deviation over the profile is $0.51 \mathrm{~mm} / \mathrm{s},(0.34 \%)$. There is, thus, no significant increase in standard deviation and bias, due to an increased axial velocity component.

\section{Flow Phantom Measurements}

The performance of the method was experimentally studied in a water bath. A recirculating flow rig consisting of a pump (Smedegaard EcoWatt 1) with a reduction valve, and a tube was used for generating the flow. The flow entered a heat-shrinking tube with a diameter of $17 \mathrm{~mm}$ after going through $1 \mathrm{~m}$ of a 20 -mm-diameter steel tube to ensure a nonturbulent parabolic velocity profile. The heatshrinking tube was submerged in water as shown in Fig. 6 and a transducer fixture ensured a precise alignment of the transducer with respect to angle and distance to the tube. A blood-mimicking fluid made by Danish Phantom Design (Jyllinge, Denmark), consisting of water, glycerol, orgasol, 


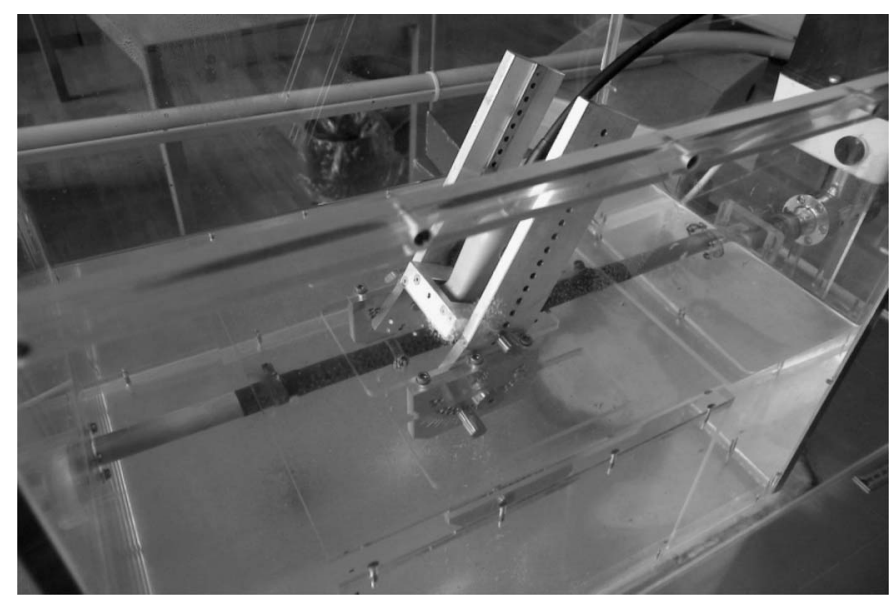

Fig. 6. Flow rig system used for the measurements. The transducer is mounted in the holder, where both angle and depth can be accurately set. The black tube is made of heat-shrink tubing and attached to steel tubes.

Trition $\mathrm{x}-100$, NaBenzoat, and $\mathrm{K}_{2}$ EDTA diluted 10 to 1 with demineralized water, was used. A $7-\mathrm{MHz}$ commercial linear array transducer was used in the experiment along with the RASMUS experimental ultrasound scanner [21]. The scanner shown in Fig. 7 can emit arbitrary signals in 128 individual channels and it can simultaneously measure 64 receive channels at $40 \mathrm{MHz}$ and 12 bits resolution. Two receive transducer elements are connected to the same receiving channel through a 2 -to- 1 multiplexer, which makes it possible to cover 128 transducer elements in 2 emissions. The data are stored in the system's 16 GBytes of memory in real-time, and they are subsequently transferred to a Linux computer cluster with 32 CPUs for beamformation and velocity estimation.

The measurements were performed using the same setup as for the simulations, with two exceptions. A $40-\mathrm{MHz}$ sampling frequency was used and a frequencyencoded chirp was used in transmission to increase the signal-to-noise ratio [22]. The chirp had a duration of $20 \mu \mathrm{s}$ and a bandwidth of $7 \mathrm{MHz}$ centered around the transducer center frequency. The chirp was weighted with a Tukey window, and a mismatched filter was used to ensure low temporal side lobes after compression, as described in [22]. The 64 receiving elements were multiplexed to be closest to the emitting center element and all 128 receiving elements were thereby sampled. The transmission was repeated with $f_{\text {prf }}=3 \mathrm{kHz}$, and 3,000 individual emissions were acquired by repeating the sequence 375 times. A summary of the parameters used for data acquisition and processing is shown in Table I.

The received RF signals were initially filtered to compress the signals in the axial direction and increase the signal-to-noise ratio [22]. The resulting signals were focused in direction of the flow, forming low-resolution directional signals. The high-resolution signals were made by adding the latest 8 low-resolution signals. Stationary echo canceling was done by finding the mean value of the directional signals and then subtracting this from the signals.

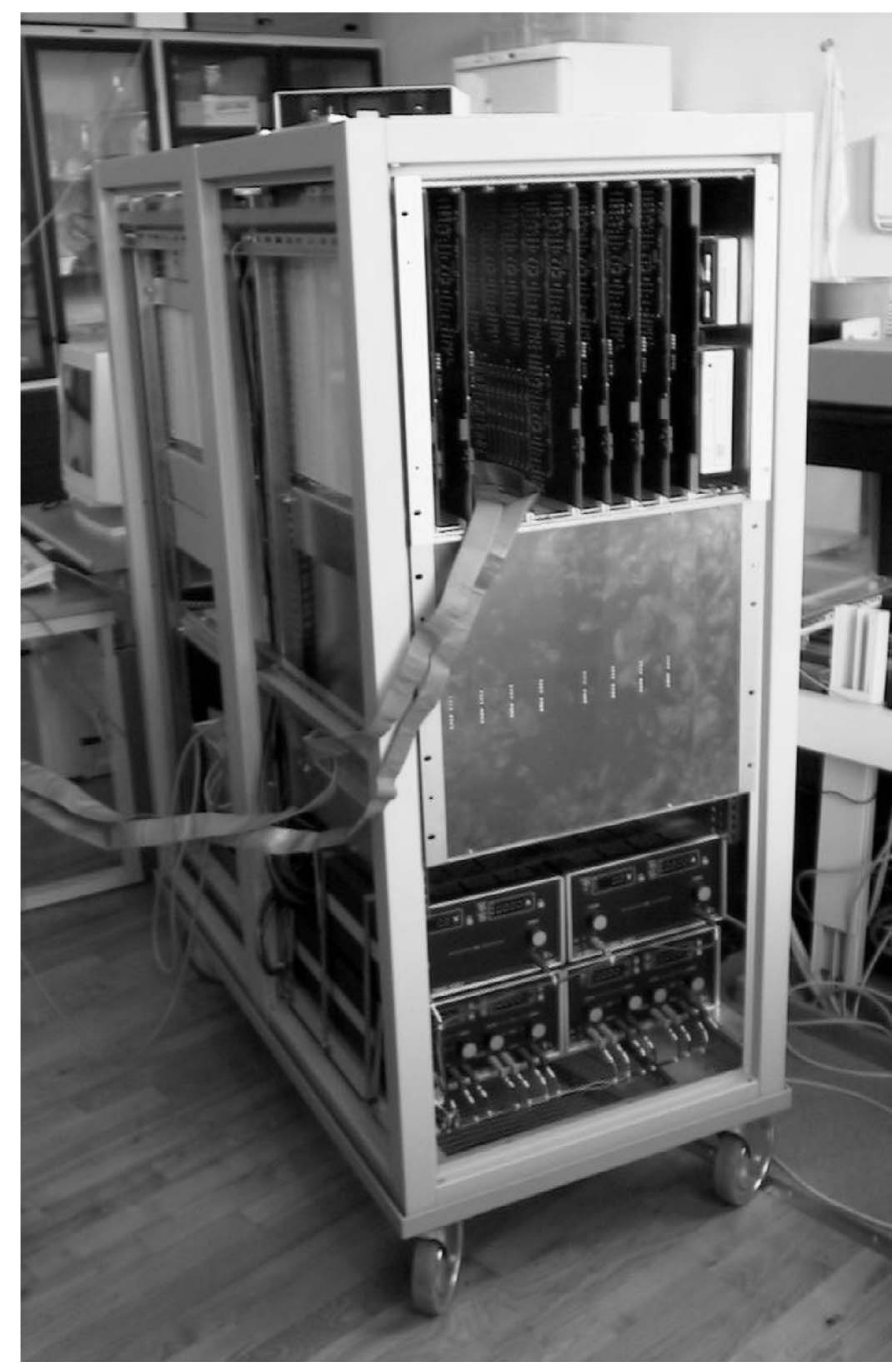

Fig. 7. The experimental ultrasound scanner RASMUS used for the experiments. The digital part of the system is shown with the 64 receivers in the top cabinet, the 128 transmitters in the middle, and the analog power supplies on the bottom. The analog front end and transducer plug are at the other side of the 19-inch racks.

The focused directional signals from the same image formation sequence were subsequently cross-correlated and added to the other cross-correlation functions. The velocity was finally found from the combined cross-correlation function using (11).

Two experiments at flow angles of 60 degrees and 90 degrees (transverse velocity) were performed. The velocity was found from 16 sequences of 8 emissions corresponding to a total of 128 emissions. This is the same number of emissions that is used in normal spectral velocity imaging [23] over which the flow normally can be considered quasistationary in the human body.

The resulting velocity profiles are shown in Fig. 8 for a flow angle of 60 degrees. Twenty independent profiles are shown on the top and the mean of the profiles \pm 3 standard deviations are shown on the bottom. It can be seen that the tube was not exactly round and a single false velocity peak was detected [23]. The relative standard deviation averaged over the profile is $0.36 \%(0.65 \mathrm{~mm} / \mathrm{s})$ compared 

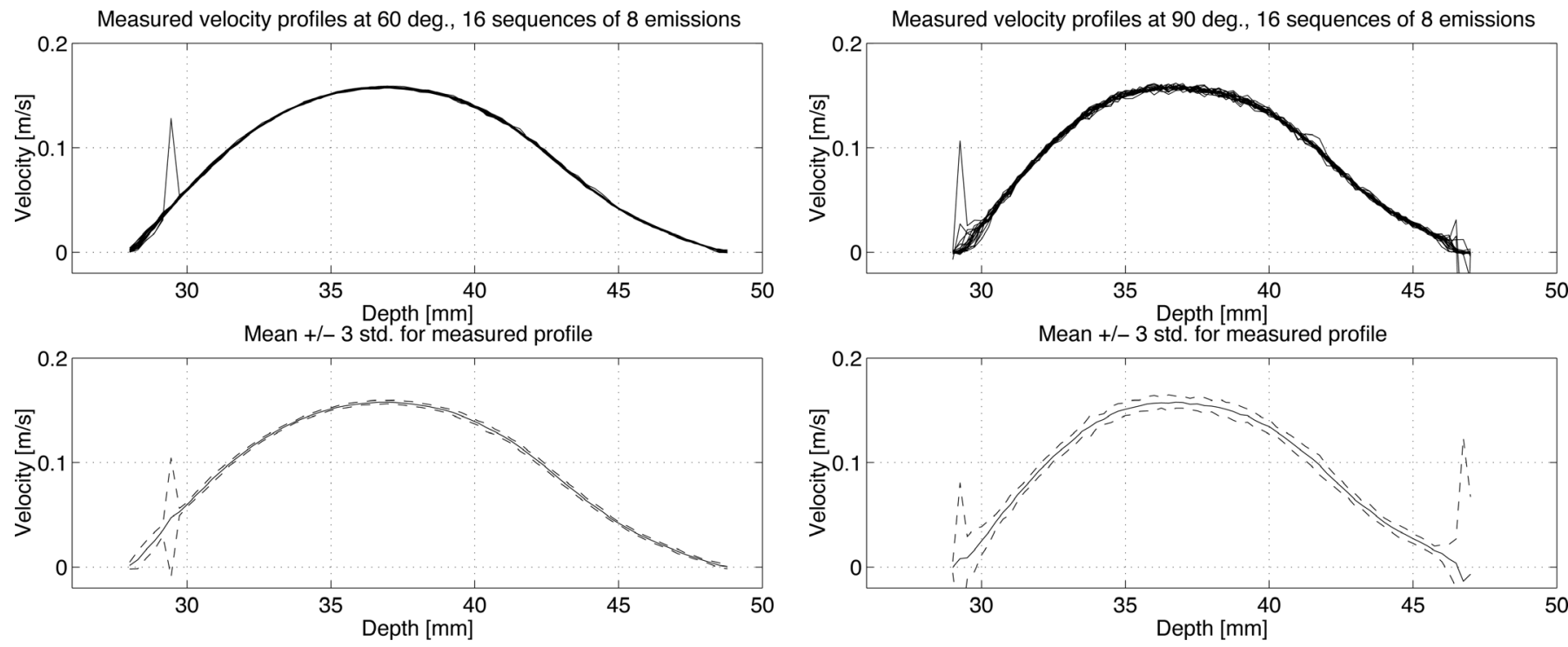

Fig. 8. Estimated profiles from the flow rig at a 60-degree flow angle. The top graph shows 20 independent profiles estimated and the bottom graph shows the mean profile (solid line) \pm 3 standard deviations (dashed lines).

to the peak velocity in the vessel, when the single false peak is neglected. This is on the same order of magnitude as for the simulated data.

The same information for 90 degrees (transverse flow) is shown in Fig. 9. Here the relative standard deviation is $2.8 \%(3.6 \mathrm{~mm} / \mathrm{s})$ compared to the peak velocity in the vessel. The accuracy is lower, but it should be recalled that traditional scanners cannot detect a transverse flow. Larger errors are seen at the edge of the vessel, and this is due to the low signal-to-noise ratio after stationary echo canceling here. Taking the standard deviation between a depth of only 30 to $45 \mathrm{~mm}$ gives a value of $1.2 \%$ $(2.1 \mathrm{~mm} / \mathrm{s})$.

\section{Parameter VARiations}

The influence of the various parameters used in the method is investigated in this Section. The relative standard deviation of the estimates is very low, as demonstrated in the previous Section, and the reference profile to be used for comparison must consequently be very accurate. The flow rig is equipped with a mass flow meter, and the velocity profile can be calculated from this, if the vessel is assumed perfectly round. Unfortunately the vessel was not perfectly round, and this resulted in a velocity profile that is not fully parabolic. A reference profile was therefore calculated based on the full set of data. The velocity was calculated from 128 emissions and all the profiles were averaged to give the mean profile shown in Fig. 10, with the profile at 60 degrees on the top and the 90-degree profile at the bottom. The slight skewness at the deep end of the vessel is easily observed. These profiles will be used as the correct profiles for all the parameter studies in this Section.

Fig. 9. Estimated profiles from the flow rig at a 90-degree flow angle. The top graph shows 20 independent profiles estimated and the bottom graph shows the mean profile (solid line) \pm 3 standard deviations (dashed lines).
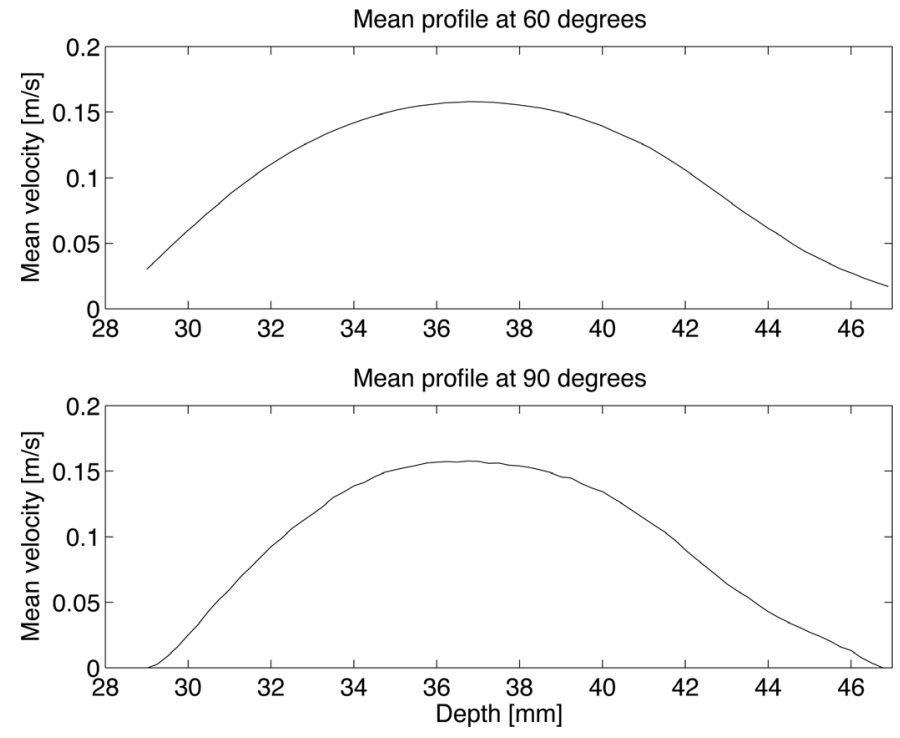

Fig. 10. Mean profiles for a 60-degree flow angle (top) and for a 90-degree flow angle (bottom).

Data for velocity estimation are continuously available for all places in the image. It is, thus, possible to make stationary echo canceling schemes that avoid the initialization effects in normal color flow imaging, and the number of directional signals used in the echo canceling and for the estimation can be different. Two examples of a variation in the number of directional signals used are shown in Figs. 11 and 12. In the first figure, only the directional signals used in the estimation are used for the echo canceling. The canceling is done by finding the mean value of all directional signals and then subtracting this mean signal from all directional signals. In Fig. 12, 128 directional signals are always used for the echo canceling regardless of 

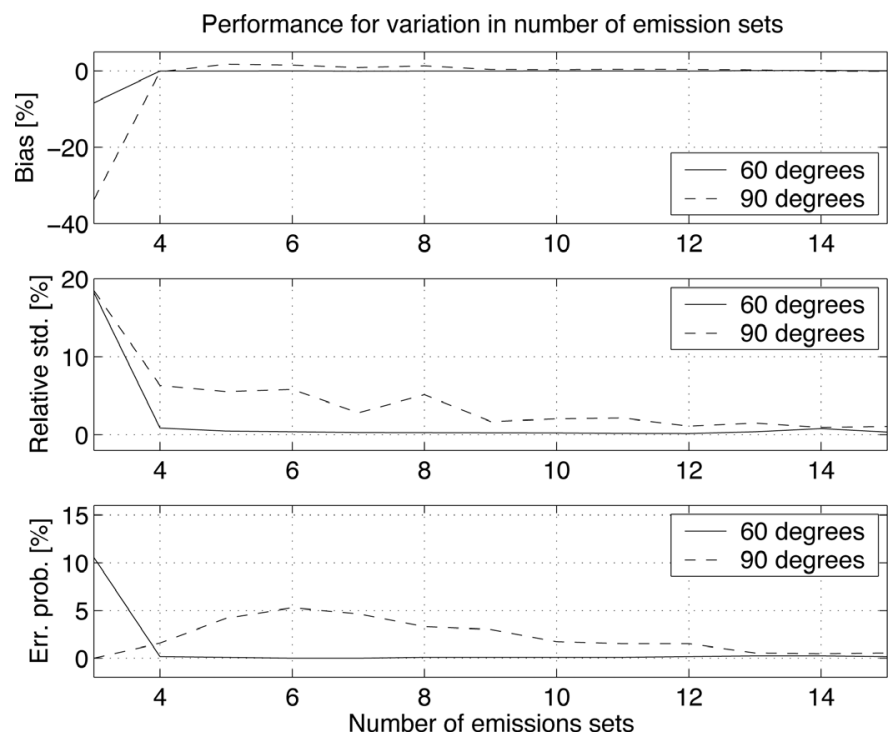

Fig. 11. Variation in the number of emissions used for the estimation, when echo canceling is done for the same number of emission sets. The solid lines show the performance for a 60-degree flow angle and the dashed lines for a 90-degree flow angle. For each angle the bias (top), standard deviation (middle), and probability of wrong detection (bottom) are shown.

how many directional signals are used for the estimation. Note that the $y$-axis scaling is different in the two figures.

Fig. 11 shows the performance as a function of number of emissions sets. Each set consists of 8 emissions with subsequent beamformation. Using 10 emission sets, thus, results in 80 directional signals. The other parameters used for the estimation can be seen in Table I. The solid line graphs show the performance at a flow angle of 60 degrees, while the dashed lines are for 90 degrees. The top graph shows the bias of the estimates compared to the reference profile averaged over the profile. The middle graph is the relative standard deviation averaged over the profile relative to the peak velocity of $0.15 \mathrm{~m} / \mathrm{s}$. The bottom graph shows the probability of false detection. An estimate is characterized as being in error when it has a value beyond the mean of all estimates \pm 5 standard deviations. The performance has in all cases been calculated for 20 estimates of the velocity profiles.

Comparing Figs. 11 and 12, it can be seen that using a few lines in this echo canceling scheme gives a much worse performance than using 16 emission pairs. This is noticeable for 3 emission pairs at 60 degrees and up to 10 emission pairs at 90 degrees. The graphs in Fig. 11 are, thus, much more influenced by the echo canceling than the actual estimation. Since this paper concentrates on the velocity estimation method, rather than the echo canceling procedure, it has been chosen to use data that have been echo canceled using 16 emission pairs for the remaining figures. More advanced echo canceling methods could also have been used.

At 60 degrees the performance shown in Fig. 12 quickly attains a satisfactory level after using 3 to 4 emission sets corresponding to 24 to 32 emissions. The probability of
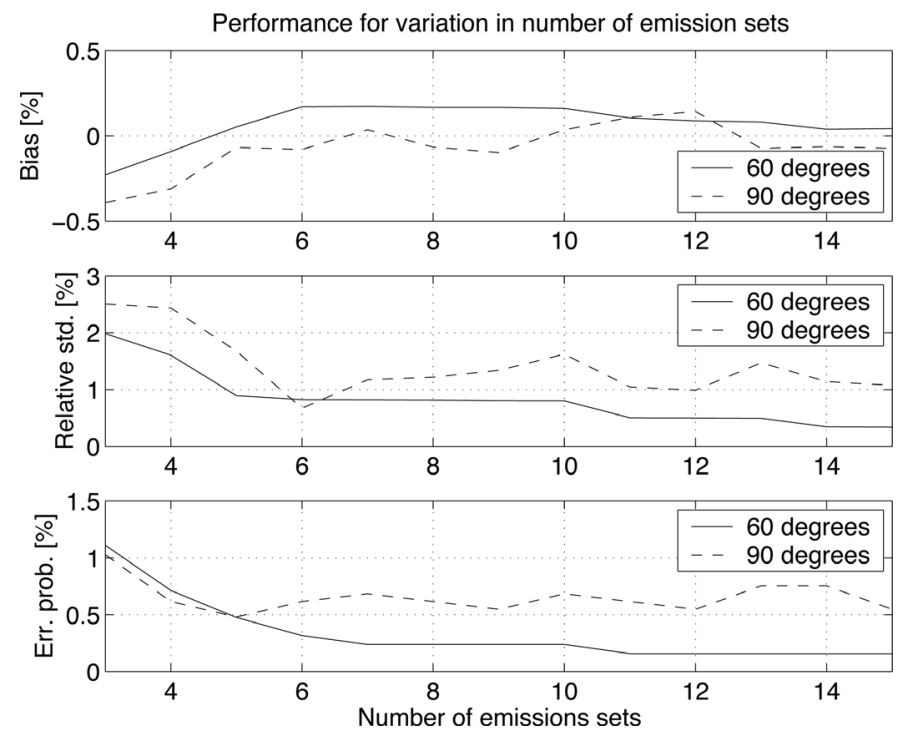

Fig. 12. Variation in the number of emissions used for the estimation, when echo canceling is done using 16 emission sets. The solid lines show the performance for a 60-degree flow angle and the dashed lines for a 90-degree flow angle. For each angle the bias (top), standard deviation (middle), and probability of wrong detection (bottom) are shown. Note that the axes are different from those in Fig. 11.

false detection falls until 6 emission sets, after which all performance indices stay relatively constant. For 90 degrees the same pattern is seen. Here the relative standard deviation falls until 6 emission sets, after which no real improvement is seen in any of the indices. It is, thus, possible to attain a satisfactory performance even for a purely transverse flow by using 24 to 48 emissions from which a full color flow image can be made. These experiments were made with a $f_{p r f}$ of $3 \mathrm{kHz}$, but they could have been made with a $f_{\text {prf }}$ up to $15 \mathrm{kHz}$, when sampling to a depth of $5 \mathrm{~cm}$. This would produce more than 310 color flow images a second. Another alternative is to use more emissions for each high-resolution image, instead of the 8 emissions used here. Having more emissions in a set will lower the side lobe level. For the higher pulse repetition frequency, 5 times more emissions could be used for each set of emissions. Then it would be possible to use 40 different emissions in each set, whereby the image quality in terms of side lobes and resolution could be significantly enhanced.

In the remaining graphs in this Section, 32 directional signals are used for the estimation corresponding to 4 sets of 8 emissions. The performance for a variation in the length of the directional signals is shown in Fig. 13. The range has been varied from $2 \lambda$ to $20 \lambda$. For 60 degrees the performance increases until a range of $6 \lambda$ to $8 \lambda$, after which no major improvement is seen. For 90 degrees the performance significantly increases until $12 \lambda$, whereafter the performance is only moderately improved until $20 \lambda$. The difference between the two angles is probably due to the different pulse shapes for the two angles. At 60 degrees the basic axial pulse is dominating, and covering just one pulse length is sufficient to give a good estimate. At 90 degrees the lateral shape of the spatial impulse response 

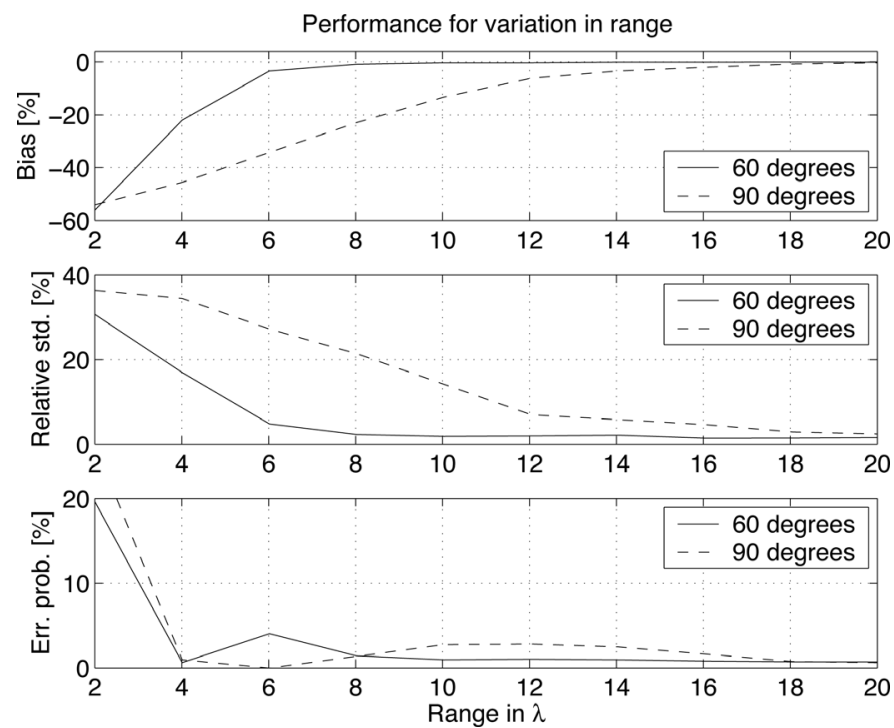

Fig. 13. Variation in the range of the directional signals used for the estimation. The solid lines show the performance for a 60-degree flow angle and the dashed lines for a 90-degree flow angle. For each angle the bias (top), standard deviation (middle), and probability of wrong detection (bottom) are shown.

is dominating, and this is longer than the axial pulse. A large range is therefore needed to cover the main part of the lateral pulse.

The spatial sampling interval $\Delta x^{\prime}$ for the directional lines was varied in Fig. 14. The interval is expressed as a fraction of $\lambda$. At 90 degrees the influence of the sampling interval is nearly negligible. A slight improvement is seen for smaller intervals in all indices. At 60 degrees the performance stays constant until the interval gets above 0.25 $\lambda$, which should be the limit.

The variation in performance for a deviation from the correct angle is shown in Fig. 15, where the $x$-axis denotes the deviation from the correct angle. At 60 degrees the bias directly follows the angle deviation between \pm 5 degrees, and the standard deviation and probability of error are only slightly affected. After this range the error rises rapidly. For 90 degrees the correct result is very dependent on the correct angle. Only a slight deviation from the correct angle will increase both the standard deviation and the bias to an unacceptable level. The probability of error is not reliably determined here, since the standard deviation is so high.

\section{Linear Array Color Flow Images}

The profiles displayed in the previous section were made using up to 128 emissions, which is considerably more than the traditional 8 to 16 emissions. The difference is, however, that a full color flow image can be made from the same data, since the received signal can be focused at all places in the image for the same set of emissions. An example of this for the 60-degree flow data is shown in Fig. 16, when a total of 128 emissions were used. The color scale
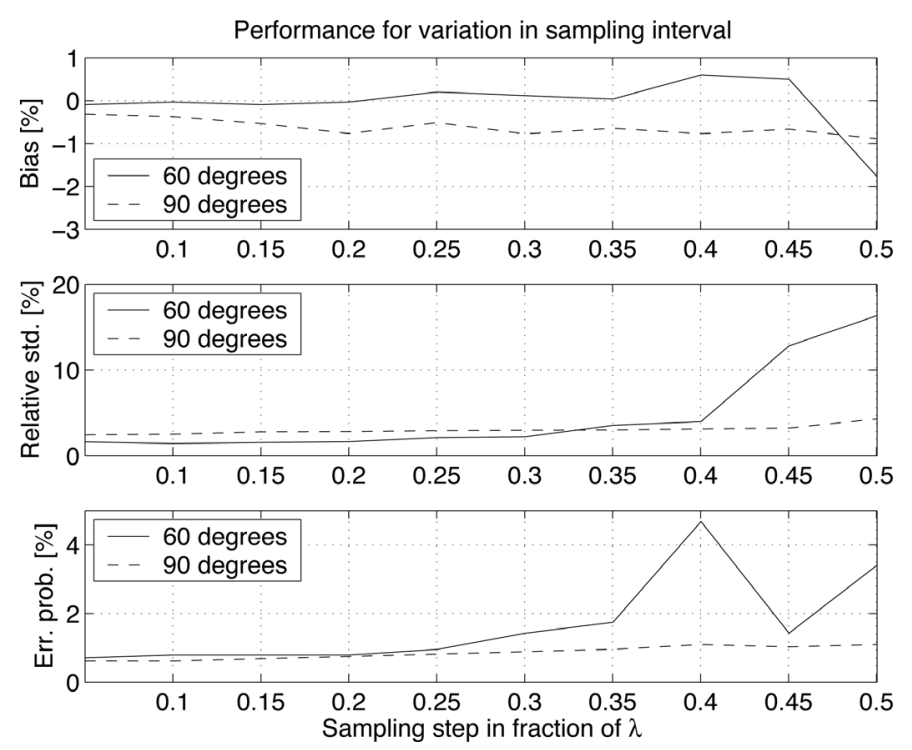

Fig. 14. Variation in the sampling interval for the directional signals used for the estimation. The solid lines show the performance for a 60-degree flow angle and the dashed lines for a 90-degree flow angle. For each angle the bias (top), standard deviation (middle), and probability of wrong detection (bottom) are shown.

indicates the velocity along the flow direction, where red hues indicate forward flow and blue reverse flow. The intensity of the color indicates the velocity magnitude.

The received data have been beamformed to make what corresponds to a linear array scan. The profiles are found with the same accuracy as the one in Fig. 8. Estimates outside the vessel are set to zero by considering the signal energy after the stationary echo canceling compared to the noise level in the measurement system. If the signal is below the noise level, the velocity estimate is set to zero. It should also be noted that no image processing has been employed on the displayed image and only the actual estimates are shown.

The second example for the 90-degree flow data is shown in Fig. 17. The full image is again made from 128 emissions, and it shows that fully transverse flow can be accurately detected using this method. A ghost flow image is seen beyond $50 \mathrm{~mm}$. This is due to re-reflections between the vessel wall and the transducer. It can possibly be eliminated by a more advanced tissue-flow discriminator.

\section{In Vivo Measurements}

The same setup as for the flow rig measurements was used to make an in vivo image of the jugular vein and the carotid artery. The result is shown in Fig. 18, where the color scale indicates the velocity along the flow direction. A red hue indicates forward flow and blue reverse flow. The 20- $\mu$ s chirp employed has a length corresponding to $15 \mathrm{~mm}$, and the velocity can therefore not be shown above $15 \mathrm{~mm}$. The pulse repetition frequency was $3 \mathrm{kHz}$ and 128 emissions (16 groups of 8 emissions) were used for finding the velocity. 

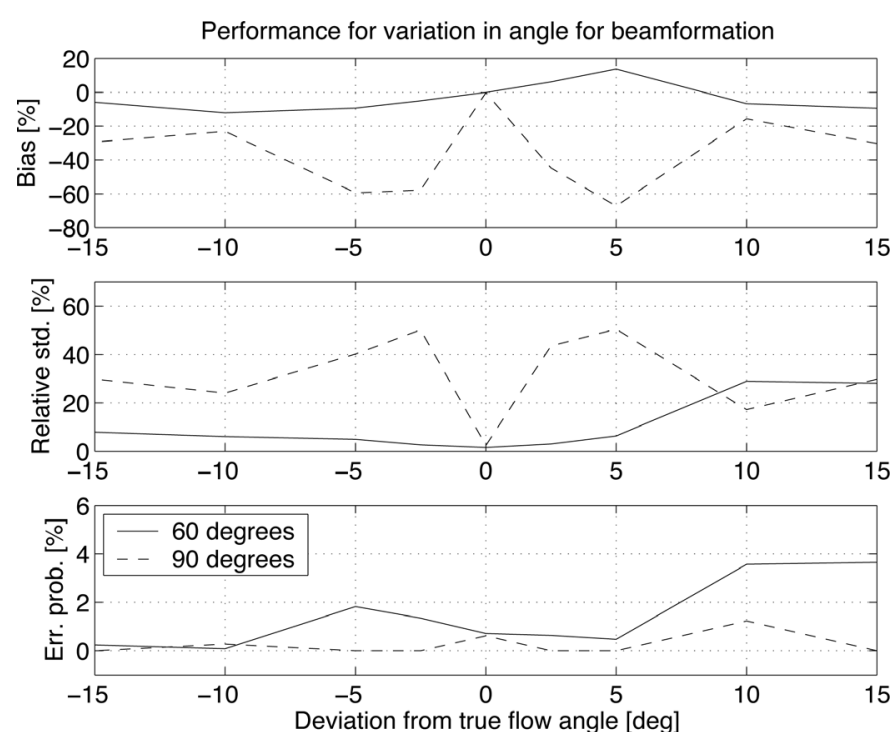

Fig. 15. Variation in angle for beamformation. The $x$-axis shows the deviation from the true flow angle. The solid lines show the performance for a 60-degree flow angle and the dashed lines for a 90-degree flow angle. For each angle the bias (top), standard deviation (middle), and probability of wrong detection (bottom) are shown.

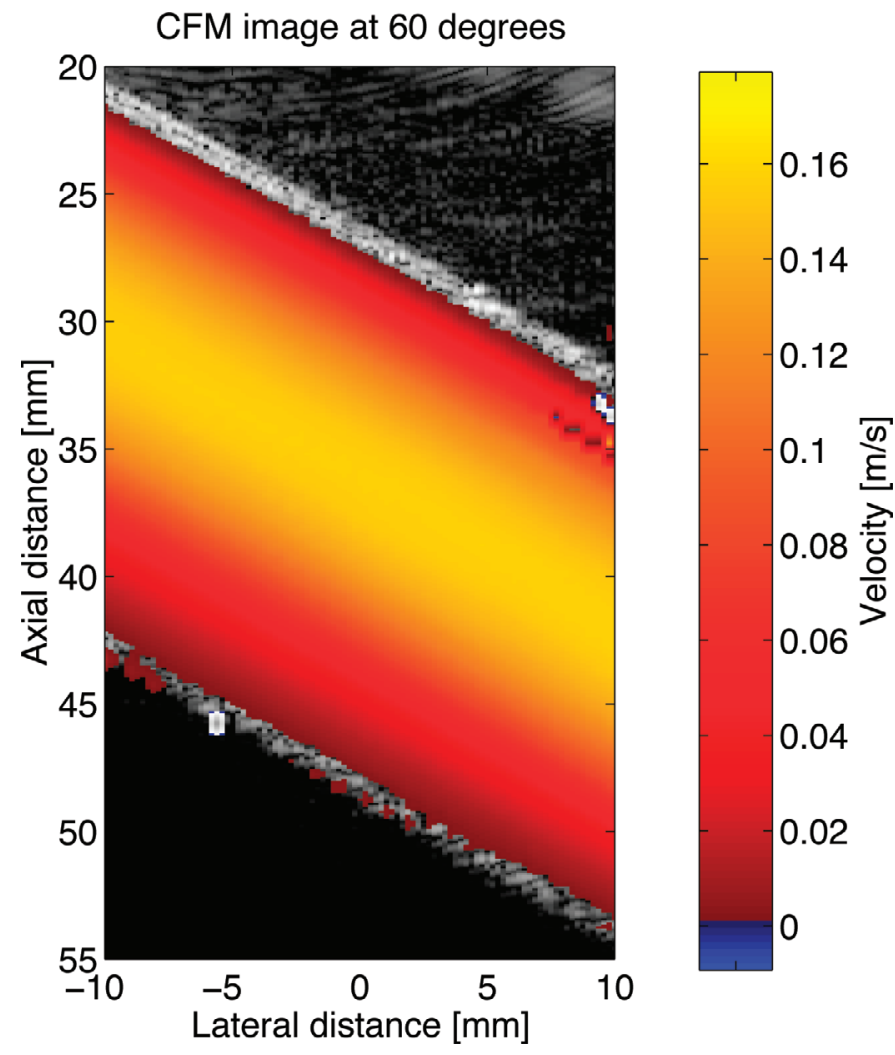

Fig. 16. Synthetic aperture color flow map image of flow rig data at a 60 -degree flow angle obtained using 128 emissions. The color scale indicates the velocity magnitude in direction of the flow.

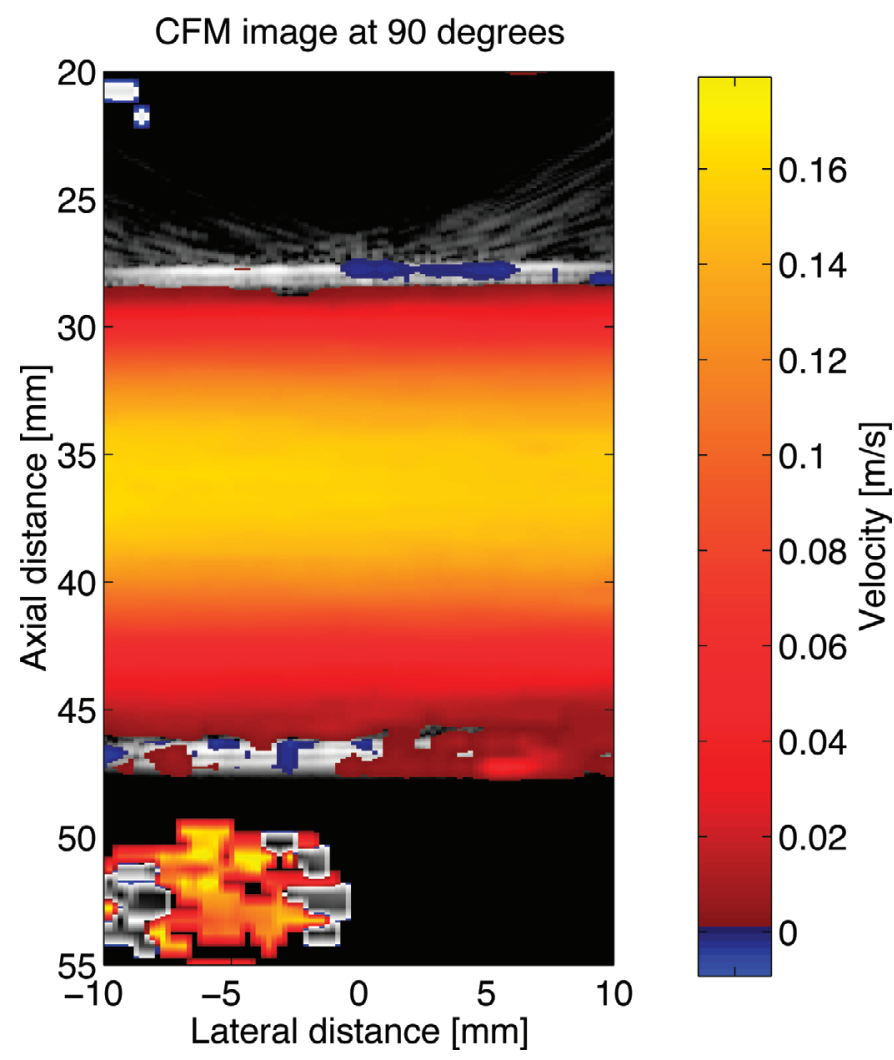

Fig. 17. Synthetic aperture color flow map image of flow rig data at a 90-degree flow angle obtained using 128 emissions.

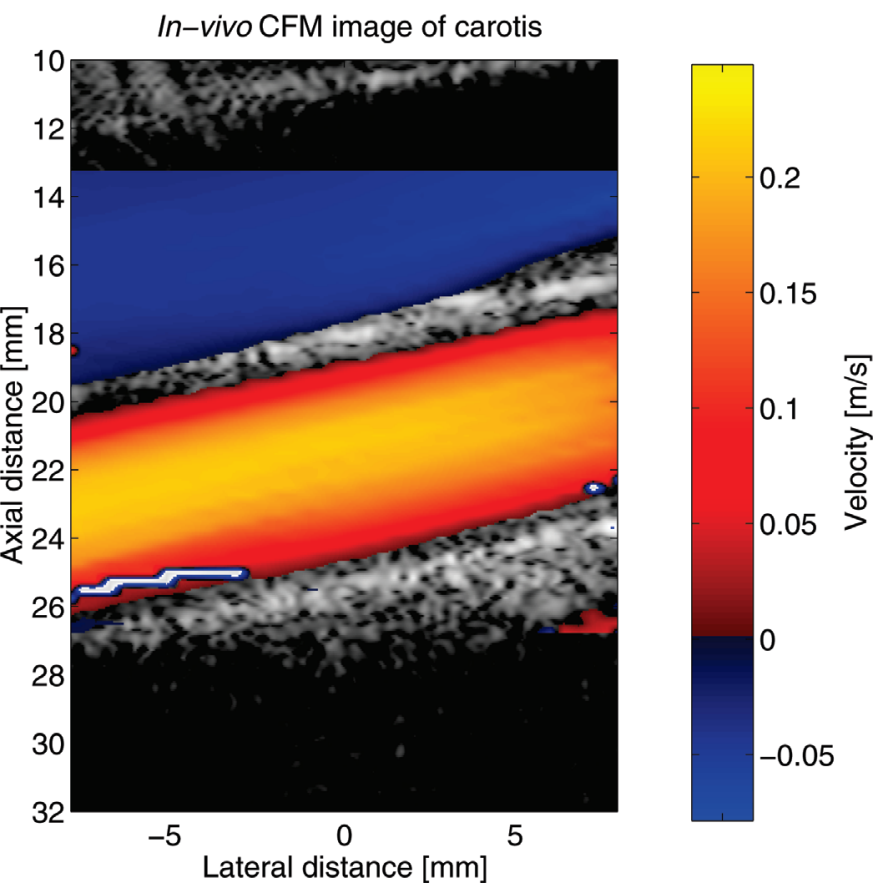

Fig. 18. In vivo color flow map image at a 77-degree flow angle for the jugular vein and carotid artery. The color scale indicates the velocity along the flow direction, where red hues indicate forward flow and blue reverse flow. 
The stationary echo canceling was changed compared to the flow rig measurements, since significant tissue motion is present in the in vivo images. A 4th-order high-pass filter was designed using the Parks-McClellan Remez routine in Matlab [24]. The stop-band was from a relative frequency of 0 to 0.04 and the pass-band amplification was 1 between 0.6 and 1. All frequencies are relative to the half sampling frequency.

The discrimination between showing the color flow image and the B-mode image was done solely on the magnitude of the velocity. It can be seen that the flow follows the vessel boundaries, and that the velocity vectors have opposite directions in the two vessels as expected. A larger velocity is seen at the center of the vessels than at the edges, which is consistent with fluid physics. A few wrong peaks can be seen at the lower edge of the carotid artery, where the signal-to-noise ratio is low, due to the stationary echo canceling.

The image is measured before the systolic phase of the cardiac cycle. At systoli there is a rapid acceleration of the flow and the method encounters problems, since the averaging of the cross-correlation functions is not valid here. We are currently investigating how to solve this problem. It should be noted that the pulse repetition frequency $f_{p r f}$ is quite low for this measurement $(3 \mathrm{kHz})$, and it can for this depth be increased to nearly $19 \mathrm{kHz}$. This would give a higher correlation between the individual measurements, and it may solve the problem.

\section{Discussion And Conclusions}

A method for making synthetic aperture flow imaging using directional focusing has been presented. The method can find the velocity magnitude of laminar flow and it can, using 32 to 128 emissions, generate a full color flow image with a standard deviation on the order of $1 \%$. It is possible to estimate velocities transverse to the ultrasound beam. The angle of the flow vectors has to be determined from the B-mode image and must currently be set manually in the estimator. This can be solved by estimating the flow angle as described in [17], where all the possible angles are investigated and the one with the highest normalized cross-correlation is selected. The angle can be different for each of the points in the image, and it is thereby possible to find velocities for different directions in the same image to correctly follow, e.g., curved vessels.

The approach has the usual advantages of synthetic aperture flow imaging: that data are continuously available. Long stationary echo canceling filters can be used, and the data available for velocity estimation are limited only by the stationarity of the flow. Very fast imaging can be attained, since the received data can be beamformed simultaneously in all directions. For an investigation depth to $5 \mathrm{~cm}$, an $f_{p r f}$ of more than $15 \mathrm{kHz}$ can be attained, and using 128 emissions per image results in a frame rate of 120 color flow images per second. Problems can, however, be experienced with an accelerating flow for moderate values of $f_{p r f}$. Solutions to this still need to be investigated. The problem is probably that the averaged cross-correlation functions are not in phase. They are therefore not summed coherently, thereby introducing errors in the peak detection, so that it is more probable to pick an erroneous peak. A possible solution is to increase $f_{\text {prf }}$ or reduce the number of emissions for the estimates. Models taking the acceleration into account can also be developed.

It should also be mentioned that the receiving elements from emission to emission can be different. This can be used to sample a large number of elements by using multiplexing as described in [25], which can be used for improving the focusing of the data in. e.g.. the elevation direction. The multiplexing also makes it possible to cover the numerous elements in a two-dimensional array to make it possible to perform three-dimensional velocity estimation as described in [26].

\section{REFERENCES}

[1] C. Kasai, K. Namekawa, A. Koyano, and R. Omoto, "Real-time two-dimensional blood flow imaging using an autocorrelation technique," IEEE Trans. Sonics Ultrason., vol. 32, pp. 458-463, 1985.

[2] O. Bonnefous and P. Pesqué, "Time domain formulation of pulse-Doppler ultrasound and blood velocity estimation by cross correlation," Ultrason. Imag., vol. 8, pp. 73-85, 1986.

[3] S. G. Foster, "A pulsed ultrasonic flowmeter employing time domain methods," Ph.D. dissertation, University of Illinois, Urbana, IL, 1985.

[4] M. D. Fox, "Multiple crossed-beam ultrasound Doppler velocimetry," IEEE Trans. Sonics Ultrason., vol. SU-25, pp. 281286, 1978.

[5] G. E. Trahey, J. W. Allison, and O. T. von Ramm, "Angle independent ultrasonic detection of blood flow," IEEE Trans. Biomed. Eng., vol. BME-34, pp. 965-967, 1987.

[6] O. Bonnefous, "Statistical analysis and time processes applied to velocity measurement," in Proc. IEEE Ultrason. Symp., 1989, pp. $887-892$.

[7] P. Munk, "Estimation of the 2-D flow vector in ultrasonic imaging: A new approach," Master's thesis, Technical University of Denmark, Lyngby, 1996.

[8] J. A. Jensen and P. Munk, "A new method for estimation of velocity vectors," IEEE Trans. Ultrason., Ferroelect., Freq. Contr., vol. 45, pp. 837-851, 1998.

[9] M. E. Anderson, "Multi-dimensional velocity estimation with ultrasound using spatial quadrature," IEEE Trans. Ultrason., Ferroelect., Freq. Contr., vol. 45, pp. 852-861, 1998.

[10] J. A. Jensen, "A new estimator for vector velocity estimation," IEEE Trans. Ultrason., Ferroelect., Freq. Contr., vol. 48, no. 4, pp. 886-894, 2001.

[11] V. L. Newhouse, D. Censor, T. Vontz, J. A. Cisneros, and B. B. Goldberg, "Ultrasound Doppler probing of flows transverse with respect to beam axis," IEEE Trans. Biomed. Eng., vol. BME-34, pp. $779-788,1987$.

[12] S. I. Nikolov and J. A. Jensen, "Velocity estimation using synthetic aperture imaging," in Proc. IEEE Ultrason. Symp., 2001, pp. 1409-1412.

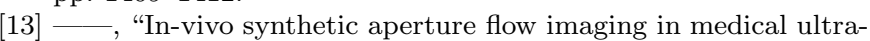
sound," IEEE Trans. Ultrason., Ferroelect., Freq. Contr., vol. 50, pp. 848-856, 2003.

[14] J. A. Jensen and I. R. Lacasa, "Estimation of blood velocity vectors using transverse ultrasound beam focusing and crosscorrelation," in Proc. IEEE Ultrason. Symp., 1999, pp. 14931497.

[15] J. A. Jensen, "Directional velocity estimation using focusing along the flow direction: I: Theory and simulation," IEEE Trans. Ultrason., Ferroelect., Freq. Contr., vol. 50, pp. 857-872, 2003. 
[16] S. I. Nikolov, K. Gammelmark, and J. A. Jensen, "Recursive ultrasound imaging," in Proc. IEEE Ultrason. Symp., vol. 2, 1999, pp. 1621-1625.

[17] J. A. Jensen, "Velocity vector estimation in synthetic aperture flow and B-mode imaging," in IEEE Int. Symp. Biomedical Imag., 2004, pp. 32-35.

[18] J. A. Jensen and N. B. Svendsen, "Calculation of pressure fields from arbitrarily shaped, apodized, and excited ultrasound transducers," IEEE Trans. Ultrason., Ferroelect., Freq. Contr., vol. 30, pp. 262-267, 1992.

[19] M. Karaman, P. C. Li, and M. O'Donnell, "Synthetic aperture imaging for small scale systems," IEEE Trans. Ultrason., Ferroelect., Freq. Contr., vol. 42, pp. 429-442, 1995.

[20] K. L. Gammelmark and J. A. Jensen, "Multielement synthetic transmit aperture imaging using temporal encoding," IEEE Trans. Med. Imag., vol. 22, no. 4, pp. 552-563, 2003.

[21] J. A. Jensen, O. Holm, L. J. Jensen, H. Bendsen, H. M. Pedersen, K. Salomonsen, J. Hansen, and S. Nikolov, "Experimental ultrasound system for real-time synthetic imaging," in Proc. IEEE Ultrason. Symp., vol. 2, 1999, pp. 1595-1599.

[22] T. X. Misaridis and J. A. Jensen, "An effective coded excitation scheme based on a predistorted FM signal and an optimized digital filter," in Proc. IEEE Ultrason. Symp., vol. 2, 1999, pp. 1589-1593.

[23] J. A. Jensen, Estimation of Blood Velocities Using Ultrasound: A Signal Processing Approach. New York: Cambridge Univ. Press, 1996.

[24] J. H. McClellan, T. W. Parks, and L. R. Rabiner, "A computer program for designing optimum fir linear phase digital filters," IEEE Trans. Audio Electroacoust., vol. AU-21, no. 6, pp. 506-526, 1973.

[25] A. T. Fernandez, K. L. Gammelmark, J. J. Dahl, C. G. Keen, R. C. Gauss, and G. E. Trahey, "Synthetic receive beamforming and image acquisition capabilities using an $8 \times 1281.75 \mathrm{D}$ array," IEEE Trans. Ultrason., Ferroelect., Freq. Contr., vol. 50, no. 1 , pp. $40-57,2003$.

[26] J. A. Jensen and S. I. Nikolov, "A method for real-time threedimensional vector velocity imaging," in Proc. IEEE Ultrason. Symp., 2003, pp. 1582-1585.

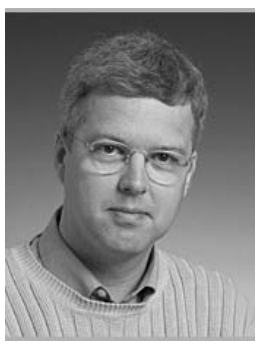

Jørgen Arendt Jensen (M'93-S'02) earned his Master of Science in electrical engineering in 1985 and the Ph.D. degree in 1989, both from the Technical University of Denmark. He received the Dr.Techn. degree from the university in 1996. He has published a number of papers on signal processing and medical ultrasound and the book Estimation of Blood Velocities Using Ultrasound, Cambridge University Press, in 1996.

He is also developer of the Field II simulation program. He has been a visiting scientist at Duke University, Stanford University, and the University of Illinois at Urbana-Champaign. He is currently full professor of Biomedical Signal Processing at the Technical University of Denmark at Ørsted•DTU and head of Center for Fast Ultrasound Imaging. He has given courses on blood velocity estimation at both Duke University and University of Illinois and teaches biomedical signal processing and medical imaging at the Technical University of Denmark. He has given several short courses on simulation, synthetic aperture imaging, and flow estimation at international scientific conferences. $\mathrm{He}$ is also the co-organizer of a new biomedical engineering education program offered by the Technical University of Denmark and the University of Copenhagen. His research is centered around simulation of ultrasound imaging, synthetic aperture imaging and blood flow estimation, and constructing systems for such imaging.

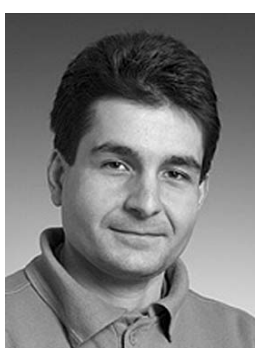

Svetoslav I. Nikolov received M.Sc. degree in electrical engineering and MBA in international business relations from the Technical University of Sofia in 1996 and 1997, respectively. In 2001 he received a Ph.D. degree from the Technical University of Denmark, Lyngby. His dissertation explored approaches for synthetic aperture tissue and flow imaging, and possibilities for real-time 3D imaging. After completing his doctoral work, he stayed on at DTU as an assistant professor, where he teaches digital design and programming. His research interests include signal and image processing and their application to ultrasound imaging. 